\title{
The Correlation of Atherosclerosis and Triglyceride Glucose Index: A Secondary Analysis of a National Cross-Sectional Study of Japanese
}

\section{xingping yang}

The First Affiliated Hospital of Guangdong Pharmaceutical University

\section{Zhao Gao}

Guangdong Sports Science Research Institute

\section{Xuming Huang}

The First Affiliated Hospital of Guangdong Pharmaceutical University

\section{Mingxing Zhang}

The First Affiliated Hospital of Guangdong Pharmaceutical University

\section{Yihua Shi}

The First Affiliated Hospital of Guangdong Pharmaceutical University

\section{Zhuoming Chen ( $\nabla$ zm_chen120@126.com )}

The First Affiliated Hospital of Jinan university $₫$ Guangdong $\rrbracket$

\section{Research}

Keywords: Triglyceride glucose index, Brachial ankle pulse wave velocity, Atherosclerosis

Posted Date: October 8th, 2021

DOl: https://doi.org/10.21203/rs.3.rs-952543/v1

License: (1) This work is licensed under a Creative Commons Attribution 4.0 International License. Read Full License 
4

5

6 Chen $^{1, *}$

$7{ }^{1}$ Department of Rehabilitation Medicine, The First Affiliated Hospital, Jinan University,

8 Guangzhou 510630, China.

$9 \quad{ }^{2}$ Department of Rehabilitation Medicine, The First Affiliated Hospital of Guangdong

10 Pharmaceutical University, Guangzhou 510080, China.

$11{ }^{3}$ Guangdong Institute of Sports Science, Guangzhou, China, Guangzhou, 510663, China.

$12 *$ Corresponding author:

13 Zhuoming Chen, Department of Rehabilitation Medicine, The First Affiliated Hospital, Jinan

14 University, No.601, West Huang pu Avenue, Guangzhou 510630, China.

15 Email: zm_chen120@126.com

17 Background: The triglyceride/glucose index (TyG index) has been suggested as a simple and 18 reliable surrogate insulin resistance (IR) marker for years. However, the association between 19 the TyG index and arterial stiffness in adults is controversial. Therefore, we evaluated this relationship, as measured based on the brachial ankle pulse wave velocity (baPWV), in 21 Japanese adults.

Methods: This study selected a total of 912 participants from the NAGALA (NAfld in Gifu Area, Longitudinal Analysis) study conducted from 2004 to 2012.The relationship between TyG index and baPWV was estimated through a logistics model. Subgroup analyses by gender, age, body mass index (BMI), total cholesterol (TC), low-density lipoprotein cholesterol (LDLC), estimated glomerular filtration rate (eGFR), and fatty liver were performed. The formula for TyG index was $\ln [1 / 2$ fasting triglyceride level $(\mathrm{mg} / \mathrm{dL}) \times$ fasting plasma glucose level $(\mathrm{mg} / \mathrm{dL})]$.

Results: After adjusting for underlying confounders, a linear relationship between TyG and baPWV was discovered. After adjusting for gender, age, body mass index, systolic blood 
31 pressure, diastolic blood pressure, high-density lipoprotein cholesterol, fatty liver, and eGFR,

32 TyG, as a continuous variable, was related to an increased risk of baPWV (adjusted odds ratio 33 [adj OR], 1.57; 95\% confidence interval [95\% CI], 1.14-2.18). Compared with the the TyG index in the first tertile, the probabilities of subjects in the third tertile that developed to baPWV were 1.78-fold higher (adj OR 1.78, 95\% CI 1.08-2.95; P for trend 0.024). Stable associations were also observed between the TyG index and baPWV in different variables through subgroup analyses.

Conclusions: The TyG index was positively and linearly related to subclinical atherosclerosis in Japanese adults and may be valuable as a predicted marker.

40 Keywords: Triglyceride glucose index, Brachial ankle pulse wave velocity, Atherosclerosis

\section{Introduction}

Cardiovascular disease (CVD) is a leading cause of death worldwide [1]. Arterial stiffening increases vascular and cardiovascular morbidity [2]. The brachial ankle pulse wave velocity (baPWV) is a candidate measure to estimate arteriosclerosis progression quantitatively noninvasively [2]. In clinical practice, the baPWV values indicate the degree of systemic arteriosclerosis and vascular disorders $[3,4]$.

Insulin resistance (IR) is among the most important risk factors for the development of arterial stiffening [5]. IR may require routine assessment in clinical practice. The homeostasis model assessment of insulin resistance (HOMA-IR) index is the most common method used to evaluate IR in clinical practice [6]. However, this method is complex, invasive, and expensive [7]. Recently, studies have demonstrated a significant correlation between the triglycerideglucose $(\mathrm{TyG})$ index (calculated as $\ln$ [fasting triglyceride $(\mathrm{mg} / \mathrm{dL}) \times$ fasting glucose $(\mathrm{mg} / \mathrm{dL}) / 2])$ and HOMA-IR [8]. The TyG index was adopted to evaluate IR with the sensitivity and specificity of $84.0 \%$ and $45.0 \%$, respectively[9]. Thus, the TyG index could serve as a simple and credible surrogate marker of IR [10-11]. Clinical approaches have traditionally been used to measure IR [12-14]. However, the relationship between the TyG index and baPWV is controversial [15-19]. In clinical practice, baPWV is used as a simple and reliable tool to measure arterial stiffness because of its high reproducibility [20-24]. 


\section{$61 \quad$ Materials and Methods}

62 Data Source

63 The secondary use of datasets was explored through the DATADRYAD database 64 (http://www.Datadryad.org/), which provides free access to the original research data (Fukuda 65 Takuya et al. (2014)) (dataset: https://doi.org/10.5061/dryad.m484p). The database provided 66 messages on variables including: gender, age, body mass index (BMI), diastolic blood pressure 67 (DBP), systolic blood pressure (SBP), alanine aminotransferase (ALT), aspartate 68 aminotransferase (AST), gamma-glutamyl transferase ( $\gamma \mathrm{GTP}$ ), fasting plasma glucose (FOG), 69 uric acid (UC), total cholesterol (TC), triglyceride (TG), high-density lipoprotein cholesterol 70 (HDL-C), low-density lipoprotein cholesterol (LDL-C), estimated glomerular filtration rate 71 (eGFR), ankle-brachial index (ABI,) brachial-ankle pulse wave velocity (baPWV).

\section{Study Population}

73 A physical examination research project from the Murakami Memorial Hospital (Gifu, Japan) 74 was performed aiming for the early detection of chronic diseases and evaluation of their 75 underlying risk factors to improve public health.

With the database, Fukuda et al. [25] reported that serum $\gamma$ GTP affected the risk of developing atherosclerosis in women. The participants underwent an examination between March 2004 and December 2012. A total of 1,445 participants (897 men and 548 women) were chosen based on the following exclusion criteria: (1) taking exogenous hormone supplementation at baseline; (2) hepatitis $\mathrm{B}(\mathrm{HBV}$ ) or $\mathrm{C}$ virus (HCV) infection; (3) fetation; and (4) ABI <0.95. A total of 912 participants (592 men and 320 women) were included in the full analysis (Figure 1). The study was approved by the ethics committee of Murakami Memorial Hospital, and all participants provided informed consent before entering the research project. 
Participants met the inclusion criteria( $(\mathrm{n}=1445)$

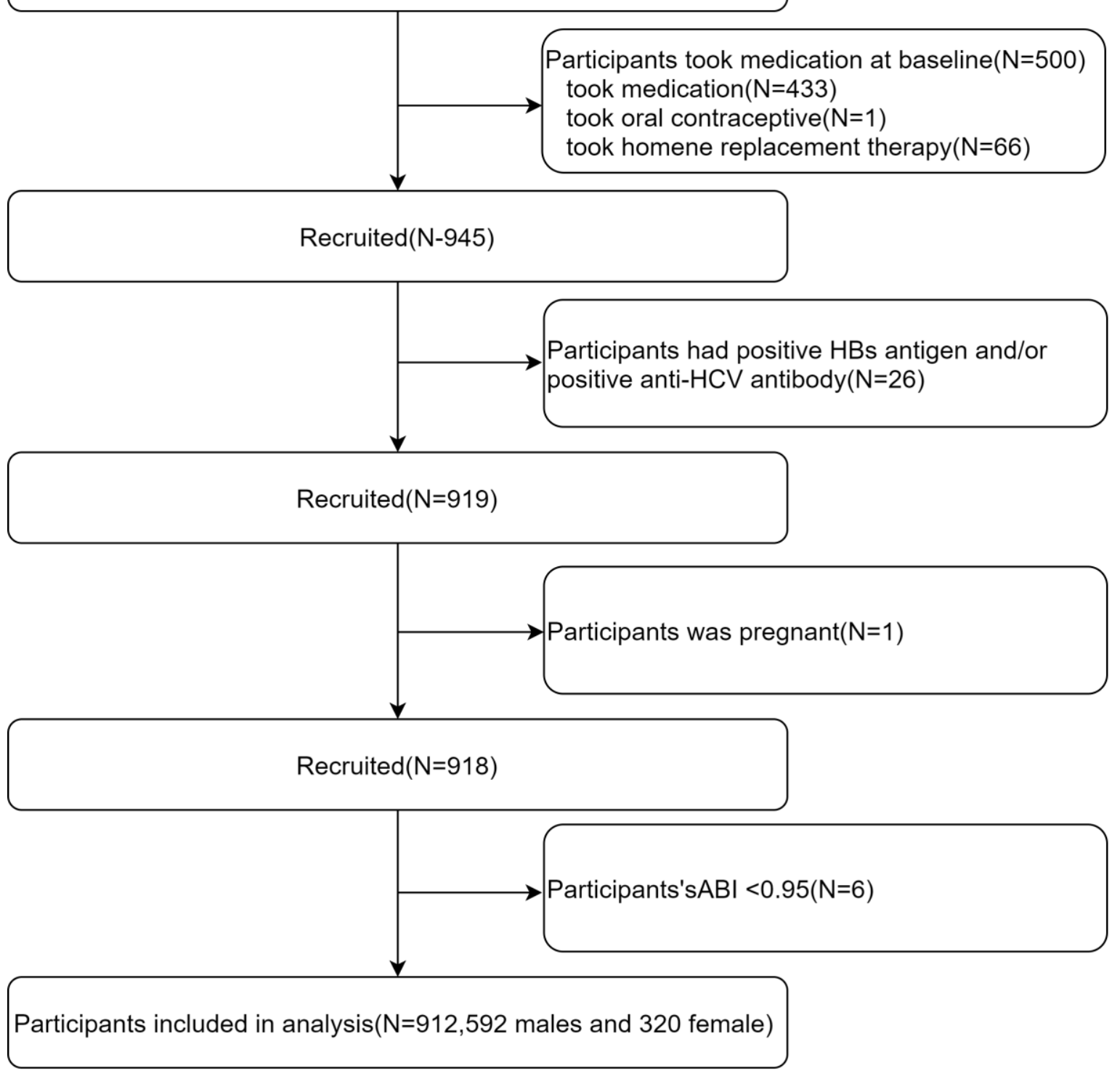

Figure 1 Flow diagram of the screening and enrollment of study participants.

Abbreviations:HBs, hepatitis B surface; $\mathrm{HCV}$,hepatitis $\mathrm{C}$ virus; $\mathrm{ABI}$,ankle-brachial index.

\section{Data Collection and Measurements}

86 The database contains information on participants' demographic characteristics, biochemical

87 indices, abdominal ultrasonography, baPWV, ABI, and lifestyle factors. Taken as an example,

88 the type of alcohol consumed and the average weekly amount of alcohol consumed during the

89 latest month were recorded and then classified into four categories: no or minimal (<40 g/week),

90 light (40-140 g/week), moderate (140-280 g/week), or heavy (> $280 \mathrm{~g} / \mathrm{week}$ ) [26]. Likewise,

91 the participants were divided into never smokers, former smokers, or current smokers. Exercise

92 that lasts long enough to cause sweating for more than 1 week, such as jogging, cycling, and 
swimming, is perceived to be normal [27]. Abdominal ultrasound detection together with artificial diagnosis by gastroenterologists were used for confirmation of suffering from fatty liver. Among the four criteria (liver/kidney echo contrast, liver luminance, deep attenuation, and vascular obscurity), subjects with liver brightness and liver/kidney contrast are judged with fatty liver disease [28]. After rested in the supine position for 5 minutes, the baPWV and ABI of the patient were measured using an automatic waveform analyzer (Colin Medical Technology, Komaki, Japan) in a room with a temperature of about $25^{\circ} \mathrm{C}$. Electrocardiogram (ECG) electrodes are placed on both wrists and the heart sound microphone is placed on the left edge of the sternum edge. The cuffs are wrapped around the arms and ankles connecting to a plethysmograph sensor that detects the form of volume pulses and an oscillating pressure sensor that determines blood pressure.

The path length from the suprasternal notch to the humerus $(\mathrm{Lb})$ and the suprasternal notch to the ankle joint (La) were determined according to the height of the subject, with the delay time from the ascending point of the brachial waveform to the ascending point of the ankle waveform (DTba) automatically determined. baPWV was assessed with the pulse wave propagation distance (Lb-La) modified with the pulse wave propagation time (DTba) divided into $\mathrm{cm} / \mathrm{s}$. [29] The reported inter-observer and inter-observer coefficients of variation were $10 \%(\mathrm{r}=0.87, \mathrm{p}<0.01)$ and $8.4 \%(\mathrm{r}=0.98, \mathrm{p}<0.01)$, respectively. The eGFR was evaluated through the formula of the Japanese Society of Nephrology (Equation 1):

$$
\mathrm{eGFR}=194 \times \mathrm{Cr}-1.094 \times \text { age }-0.287\left(\mathrm{~mL} / \mathrm{min} / 1.73 \mathrm{~m}^{2}\right)
$$

for men, and was multiplied by a correction factor of 0.739 for women [30]. The formula for the TyG index was as shown in Equation 2:

$\ln [1 / 2$ fasting triglyceride level $(\mathrm{mg} / \mathrm{dL}) \times$ fasting plasma glucose $(\mathrm{FPG})(\mathrm{mg} / \mathrm{dL})][31]$.

\section{Statistical Analysis}

Normal and skew distributions of continuous variables are expressed as mean values with standard deviation (SD) and median values with inter-quaternary interval (IQRS), respectively. Classification variables are expressed in terms of frequency and percentage. We used the chisquare test, one-way analysis of variance, or Kruskal-Wallis test to examine the statistical differences between groups stratified by the tertiles of the TyG index. Univariate and 
multivariate logistic proportional hazard model was used to examine the relationship between

126 the TyG index and the risk of baPWV. Three models were employed: model 1 as a 127 crude model: univariate; model 2 modified with age and gender; and model 3, adjusted for 128 model 2 plus BMI, SBP, DBP, HDL-C, fatty liver, and eGFR. In these models, the median 129 values in each tertile of the TyG index were used for the operation of linear trend tests. The 130 logistic relationship between the TyG and baPWV was estimated using logistic regression with 131 restricted cubic splines. For identification of modifications and interactions, subgroups 132 analyses were developed with stratified linear regression models and the likelihood ratio test 133 (LRT) regarding gender, age ( $<55$ or $\geq 55$ years), BMI ( $<25$ or $\geq 25 \mathrm{~kg} / \mathrm{m} 2)$, TC $(<208$ or $\geq 208$ $134 \mathrm{mg} / \mathrm{dL})$, LDL-C ( $<126$ or $\geq 126 \mathrm{mg} / \mathrm{dL})$, eGFR ( $<60$ or $\geq 60 \mathrm{~mL} / \mathrm{min} / 1.73 \mathrm{~m} 2)$, and fatty liver 135 (none, moderate, or severe). All analyses were performed using the statistical software 136 packages in R 3.3.2 (http://www.R-project.org, The R Foundation) and Free Statistics software 137 version 1.1. Differences with P-values $<0.05$ were considered statistically significant.

\section{Results}

\section{Baseline Characteristics of Selected Participants}

140 This cross-sectional study included a total of 912 participants. Table 1 shows the baseline 141 characteristics of the participants according to the tertiles of the TyG index. The mean age of 142 the 912 participants was $51.1 \pm 9.6$ years and $64.9 \%$ were male. Participants in the third group 143 of TyG (Q3) had higher TyG values; were more likely to be male; have higher BMI, SBP, DBP, 144 FBG, uric acid, AST, ALT, $\gamma$ GTP, TC, TG, and LDL-C levels; higher eGFR; were more likely 145 to be current smokers, consume > $280 \mathrm{~g} /$ week alcohol, exercise <1/week, more likely to have 146 moderate or severe fatty liver, and higher baPWV compared to the other groups (Q1-2).

147 Participants in the third group of TyG (Q3) had lower HDL-C and eGFR values and were more 148 likely to be none or past smokers, consume $0-40 \mathrm{~g} /$ week alcohol, exercise $\geq 1 /$ week, and have 149 no fatty liver compared to the other groups (Q1-2).

Table 1 Baseline characteristics of the study participants sorted by tertiles of TyG.

\begin{tabular}{|c|c|c|c|c|c|}
\hline & All participants & Q1(<7.96) & Q2(7.96 8.57) & $\mathrm{Q} 3(>8.57)$ & $P$ value \\
\hline Participants(n) & 912 & 304 & 304 & 304 & \\
\hline TyG index & $8.3 \pm 0.7$ & $7.6 \pm 0.3$ & $8.3 \pm 0.2$ & $9.0 \pm 0.4$ & $<0.001$ \\
\hline $\operatorname{Sex}(\mathrm{n}(\%))$ & & & & & $<0.001$ \\
\hline Males & $592(64.9)$ & $128(42.1)$ & $203(66.8)$ & $261(85.9)$ & \\
\hline Females & $320(35.1)$ & $176(57.9)$ & $101(33.2)$ & $43(14.1)$ & \\
\hline Age(years) & $51.1 \pm 9.6$ & $50.7 \pm 10.1$ & $51.6 \pm 9.4$ & $51.1 \pm 9.2$ & 0.493 \\
\hline
\end{tabular}




\begin{tabular}{|c|c|c|c|c|c|}
\hline $\operatorname{BMI}\left(\left(\mathrm{kg} / \mathrm{m}^{2}\right)\right.$ & $23.1 \pm 3.1$ & $21.8 \pm 2.5$ & $23.1 \pm 3.3$ & $24.5 \pm 3.0$ & $<0.001$ \\
\hline SBP (mmHg) & $120.2 \pm 15.0$ & $114.7 \pm 13.9$ & $120.9 \pm 15.4$ & $125.1 \pm 13.7$ & $<0.001$ \\
\hline DBP (mmHg) & $76.1 \pm 10.0$ & $72.0 \pm 9.4$ & $76.4 \pm 10.2$ & $80.0 \pm 8.7$ & $<0.001$ \\
\hline FBG (mg/dL) & $98.1 \pm 14.1$ & $92.2 \pm 7.6$ & $98.0 \pm 10.4$ & $104.0 \pm 18.9$ & $<0.001$ \\
\hline Uric $\operatorname{acid}(\mathrm{mg} / \mathrm{dL})$ & $5.3 \pm 1.4$ & $4.6 \pm 1.3$ & $5.2 \pm 1.3$ & $6.0 \pm 1.3$ & $<0.001$ \\
\hline AST(IU/L) & $19.0(16.0,23.0)$ & $19.0(16.0,22.0)$ & $19.0(16.0,23.0)$ & $21.0(17.0,26.0)$ & $<0.001$ \\
\hline ALT(IU/L) & $19.0(14.0,26.0)$ & $16.0(12.8,20.0)$ & $18.0(14.0,23.2)$ & $23.0(18.0,34.0)$ & $<0.001$ \\
\hline$\gamma \mathrm{GTP}(\mathrm{IU} / \mathrm{L})$ & $19.0(14.0,28.0)$ & $14.0(11.0,19.0)$ & $18.5(14.0,26.0)$ & $25.0(18.0,43.0)$ & $<0.001$ \\
\hline $\mathrm{TC}(\mathrm{mg} / \mathrm{dL})$ & $209.8 \pm 35.9$ & $198.4 \pm 33.9$ & $208.0 \pm 33.7$ & $223.1 \pm 35.8$ & $<0.001$ \\
\hline $\mathrm{TG}(\mathrm{mg} / \mathrm{dL})$ & $99.9 \pm 74.9$ & $44.2 \pm 12.3$ & $81.8 \pm 14.8$ & $173.6 \pm 87.3$ & $<0.001$ \\
\hline $\mathrm{HDL}-\mathrm{C}(\mathrm{mg} / \mathrm{dL})$ & $53.5 \pm 14.6$ & $62.0 \pm 14.0$ & $53.4 \pm 13.1$ & $45.2 \pm 11.4$ & $<0.001$ \\
\hline $\mathrm{LDL}-\mathrm{C}(\mathrm{mg} / \mathrm{dL})$ & $128.1 \pm 31.7$ & $116.6 \pm 29.2$ & $130.4 \pm 28.7$ & $137.1 \pm 33.5$ & $<0.001$ \\
\hline $\mathrm{eGFR}\left(\mathrm{mL} / \mathrm{min} / 1.73 \mathrm{~m}^{2}\right)$ & $70.4 \pm 12.0$ & $73.5 \pm 13.0$ & $70.2 \pm 11.4$ & $67.6 \pm 11.0$ & $<0.001$ \\
\hline Current smoker (n (\%)) & & & & & $<0.001$ \\
\hline None or past & $715(78.4)$ & $263(86.5)$ & $233(76.6)$ & $219(72)$ & \\
\hline Current & $197(21.6)$ & $41(13.5)$ & $71(23.4)$ & $85(28)$ & \\
\hline Alcohol group (n (\%)) & & & & & $<0.001$ \\
\hline $0-40 \mathrm{~g} /$ week & $594(65.1)$ & $225(74)$ & $194(63.8)$ & 175 (57.6) & \\
\hline $40-140 \mathrm{~g} /$ week & $150(16.4)$ & $42(13.8)$ & $59(19.4)$ & $49(16.1)$ & \\
\hline 140-280g/week & $88(9.6)$ & $23(7.6)$ & $33(10.9)$ & $32(10.5)$ & \\
\hline more than $280 \mathrm{~g} /$ week & $80(8.8)$ & $14(4.6)$ & $18(5.9)$ & $48(15.8)$ & \\
\hline Regular exercise (n (\%)) & & & & & 0.008 \\
\hline$<1 /$ week & $735(80.6)$ & $229(75.3)$ & $247(81.2)$ & $259(85.2)$ & \\
\hline$\geq 1 /$ week & $177(19.4)$ & $75(24.7)$ & $57(18.8)$ & $45(14.8)$ & \\
\hline Fatty liver (n (\%)) & & & & & $<0.001$ \\
\hline None & 647 (70.9) & $274(90.1)$ & $229(75.3)$ & $144(47.4)$ & \\
\hline Moderate or severe & $265(29.1)$ & $30(9.9)$ & $75(24.7)$ & $160(52.6)$ & \\
\hline Menopausal state (n (\%)) & & & & & 0.071 \\
\hline Menopausal & $138(43.1)$ & $86(48.9)$ & $36(35.6)$ & $16(37.2)$ & \\
\hline Postmenopausal & $182(56.9)$ & $90(51.1)$ & $65(64.4)$ & $27(62.8)$ & \\
\hline ABI & $1.2 \pm 0.1$ & $1.2 \pm 0.1$ & $1.2 \pm 0.1$ & $1.2 \pm 0.1$ & 0.001 \\
\hline $\mathrm{baPWV}(\mathrm{cm} / \mathrm{s})$ & $1415.8 \pm 246.3$ & $1350.7 \pm 226.1$ & $1424.5 \pm 234.6$ & $1472.1 \pm 262.0$ & $<0.001$ \\
\hline
\end{tabular}

Notes: Values are expressed as mean \pm standard deviation or $\mathrm{n}(\%)$. Abbreviations: TyG index, triglyceride glucose index; BMI, body mass index; SBP, systolic blood pressure; DBP, diastolic blood pressure; FPG, fasting plasma glucose; AST, aspartate aminotransferase; ALT, alanine aminotransferase; $\gamma \mathrm{GTP}$, gamma-glutamyl transferase; TC, total cholesterol; TG, triglyceride; HDL-C, high-density lipoprotein cholesterol; LDL-C, low-density lipoprotein cholesterol; eGFR: estimated glomerular filtration rate; ABI, ankle-brachial index; baPWV, brachial to ankle pulse wave velocity. 
151 Table 2 presents the univariate analysis results of the relationship between risk factors and

152 baPWV. The univariate logistics model showed that BMI, HDL-C level, current smoker,

153 regular exercise, and ABI were not associated with baPWV. In contrast, gender, age, SBP,

154 DBP, FBG, uric acid, AST, ALT, $\gamma$ GTP, TC, TG, TyG, and LDL-C levels, eGFR, alcohol

155 use, fatty liver, and menopausal state were positively related to baPWV.

Table 2 Univariate analysis of baPWV.

\begin{tabular}{|c|c|c|}
\hline & OR $(95 \% \mathrm{CI})$ & $P$ value \\
\hline $\operatorname{Sex}(n(\%))$ & & 0.035 \\
\hline Males & Ref. & \\
\hline Females & $0.74(0.56,0.98)$ & \\
\hline Age(year) & $1.10(1.09,1.12)$ & $<0.001$ \\
\hline BMI $\left(\mathrm{kg} / \mathrm{m}^{2}\right)$ & $1.04(1.00,1.08)$ & 0.069 \\
\hline $\mathrm{SBP}(\mathrm{mmHg})$ & $1.07(1.06,1.09)$ & $<0.001$ \\
\hline $\mathrm{DBP}(\mathrm{mmHg}$ & $1.10(1.09,1.12)$ & $<0.001$ \\
\hline $\mathrm{FBG}(\mathrm{mg} / \mathrm{dL})$ & $1.04(1.02,1.05)$ & $<0.001$ \\
\hline Uric $\operatorname{acid}(\mathrm{mg} / \mathrm{dL})$ & $1.14(1.04,1.26)$ & 0.007 \\
\hline AST(IU/L) & $1.03(1.01,1.04)$ & 0.004 \\
\hline ALT(IU/L) & $1.01(1.00,1.02)$ & 0.024 \\
\hline$\gamma \mathrm{GTP}(\mathrm{IU} / \mathrm{L})$ & $1.01(1.00,1.02)$ & 0.001 \\
\hline $\mathrm{TC}(\mathrm{mg} / \mathrm{dL})$ & $1.01(1.00,1.01)$ & $<0.001$ \\
\hline TG (mg/dL) & $1.00(1.00,1.01)$ & $<0.001$ \\
\hline TyG index & $1.84(1.50,2.27)$ & $<0.001$ \\
\hline HDL-C(mg/dL) & $0.99(0.99,1.00)$ & 0.249 \\
\hline LDL-C(mg/dL) & $1.01(1.00,1.01)$ & 0.012 \\
\hline $\mathrm{eGFR}\left(\mathrm{mL} / \mathrm{min} / 1.73 \mathrm{~m}^{2}\right)$ & $0.96(0.94,0.97)$ & $<0.001$ \\
\hline Current smoker (n (\%)) & & 0.467 \\
\hline None or past & Ref. & \\
\hline Current & $0.89(0.65,1.22)$ & \\
\hline Alcohol group (n (\%)) & & 0.08 \\
\hline $0-40 \mathrm{~g} /$ week & Ref. & \\
\hline 40-140g/week & $0.98(0.68,1.40)$ & 0.896 \\
\hline
\end{tabular}




\begin{tabular}{|c|c|c|}
\hline $140-280 \mathrm{~g} /$ week & $1.35(0.86,2.11)$ & 0.192 \\
\hline more than $280 \mathrm{~g} /$ week & $1.73(1.08,2.77)$ & 0.022 \\
\hline Regular exercise (n (\%)) & & 0.814 \\
\hline$<1 /$ week & Ref. & \\
\hline$\geq 1 /$ week & $1.04(0.75,1.45)$ & \\
\hline Fatty liver (n (\%)) & & $<0.001$ \\
\hline None & Ref. & \\
\hline Moderate or severe & $1.92(1.44,2.56)$ & \\
\hline Menopausal state (n (\%)) & & $<0.001$ \\
\hline Menopausal & Ref. & \\
\hline Postmenopausal & $5.37(3.20,9.01)$ & $<0.001$ \\
\hline ABI & $3.20(0.49,20.72)$ & 0.222 \\
\hline
\end{tabular}

156 Unadjusted and Adjusted Logistics Models

157 Linear logistic models were adopted to evaluate the independent relationship between the TyG 158 index and baPWV (univariate and multivariate logistics models). Table 3 presents the effect 159 sizes (ORs) and 95\% confidence intervals (95\% CIs). In the crude model (model 1), a one-unit 160 increase in the TyG index was related to an $84 \%$ higher risk of incident increased baPWV (OR 161 1.84, 95\% CI 1.50-2.27). In model 2, a one-unit increase in TyG index increased the risk of 162 evolving baPWV by $91 \%$ (OR 1.91, 95\% CI 1.49-2.44) after adjusting for gender and age. In 163 model 3, each one-unit increase in TyG index was a 55\% higher risk of incident increased 164 baPWV (OR 1.57, 95\% CI 1.14-2.18). For sensitivity analysis, we transformed the TyG index 165 into a categorical variable (tertile of TyG index). The results showed that the P-value for the 166 trend of TyG index as categorical variables was concomitant with the result of the TyG index 167 as continuous variables in the different models.

Table 3 Relationship between TyG and baPWV in different models.

\begin{tabular}{ccccccc}
\hline & Model1 & P value & Model2 & P value & Model3 & P value \\
\hline TyG index & $1.84(1.50,2.27)$ & $<0.001$ & $1.91(1.49,2.44)$ & $<0.001$ & $1.57(1.14,2.18)$ & 0.006
\end{tabular}


TyG index

$\mathrm{Q} 1(<7.96)$

Ref.

Ref.

Ref.

$\begin{array}{ccccccr}\text { Q2(7.96 8.57) } & 1.74(1.25,2.42) & <0.001 & 1.66(1.15,2.41) & 0.196 & 1.43(0.93,2.19) & 0.101 \\ \text { Q3(>8.57) } & 2.42(1.74,3.36) & <0.001 & 2.50(1.70,3.71) & <0.001 & 1.78(1.08,2.95) & 0.024 \\ \text { P for trend } & <0.001 & <0.001 & & 0.024\end{array}$

Model 1 was not adjusted. Model 2 was adjusted for gender and age. Model 3 was adjusted for gender, age, BMI, SBP, DBP, HDL-C, fatty liver, eGFR. Abbreviations: BMI, body mass index; SBP, systolic blood pressure; DBP, diastolic blood pressure; HDL-C, high-density lipoprotein cholesterol; eGFR, estimated glomerular filtration rate; TyG, triglyceride glucose index; baPWV, brachial to ankle pulse wave velocity; CI, confidence interval.

\section{Threshold Effect Analysis of the TyG on Incident baPWV}

169 We used a logistics regression model with cubic spline function to assess the correlation of 170 TyG index and baPWV (Figure 2). After adjusting for gender, age, BMI, SBP, DBP, TC, LDL171 C, fatty liver, eGFR, TyG index and baPWV showed positive linear correlation.

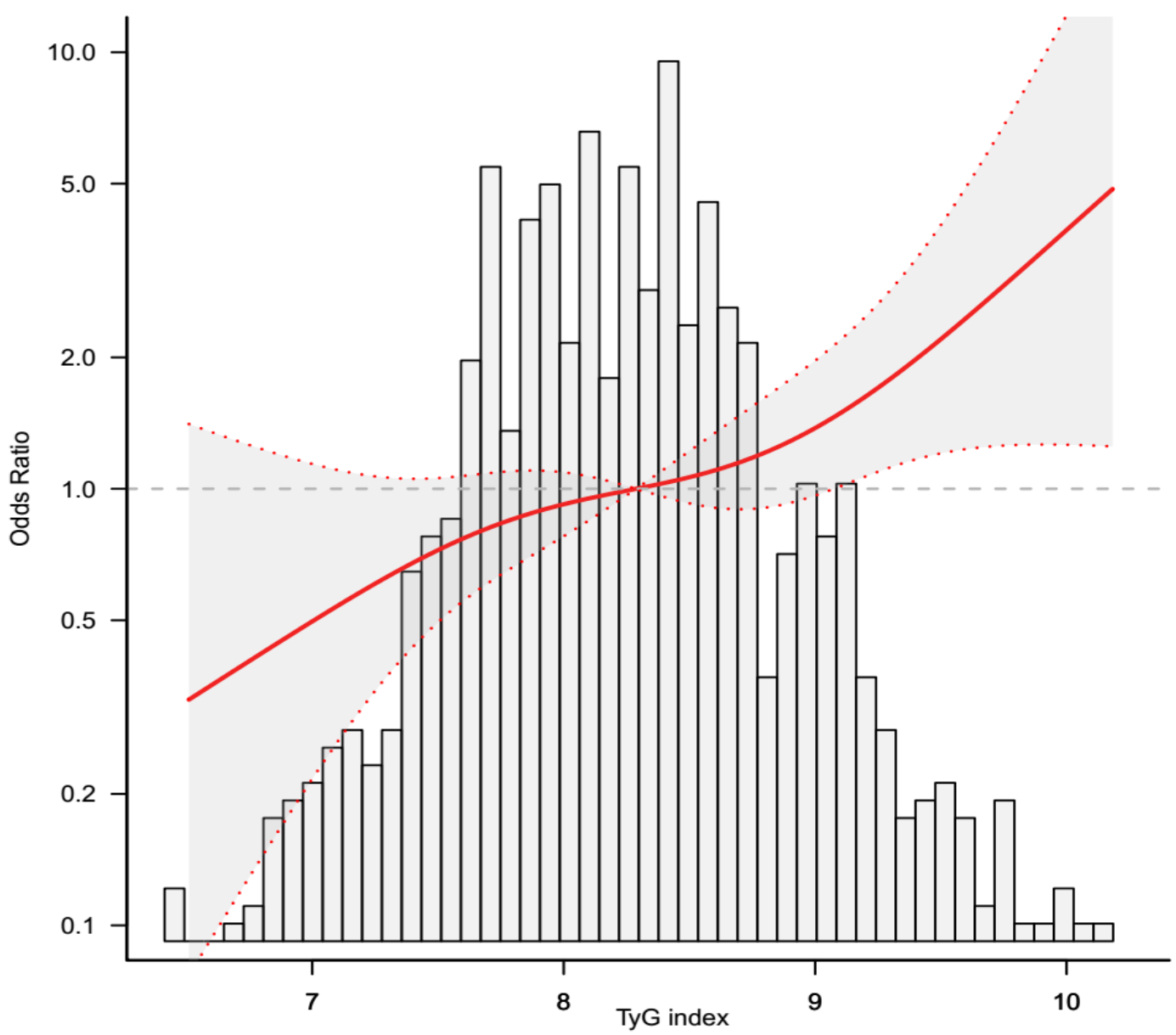

Figure 2 The relations between TyG index and baPWV 
173 The grey histograms represent the distributions between variables. The solid red curve

174 indicates the smoothing curve fit between variables. The grey zone indicates the $95 \%$

175 confidence interval of the curve fit. Data was adjusted for gender, age, BMI, SBP, DBP,

176 HDL-C, fatty liver, eGFR. Abbreviations: BMI, body mass index; SBP, systolic blood

177 pressure; DBP, diastolic blood pressure; HDL-C, High-density lipoprotein cholesterol; eGFR,

178 estimated glomerular filtration rate; TyG, triglyceride glucose index; baPWV, brachial to

179 ankle pulse wave velocity; CI, confidence interval.

\section{Subgroup Analyses}

181 The results of subgroup analyses of the association between the TyG index and baPWV are

182 indicated in Figure 3. The participants were split into subgroups and the results indicated that

183 there exists a steady relationship between TyG and incident baPWV in different subgroups.

\begin{tabular}{|c|c|c|c|c|c|}
\hline & $<1400 \mathrm{~cm} / \mathrm{s}(\mathrm{n})$ & $\geq 1400 \mathrm{~cm} / \mathrm{s}(\mathrm{n}(\%))$ & adj OR(95\%Cl) & $P$ value & $P$ for interaction \\
\hline Sex & & & & & 0.693 \\
\hline Males & 592 & $278(47.0)$ & $1.50(1.07,2.10)$ & 0.020 & \\
\hline Females & 320 & $127(39.7)$ & $1.69(1.05,2.72)$ & 0.030 & \\
\hline $\mathrm{BMI}\left(\mathrm{kg} / \mathrm{m}^{2}\right)$ & & & & & 0.988 \\
\hline$<25$ & 690 & $301(43.6)$ & $1.62(1.17,2.24)$ & 0.004 & \\
\hline$\geq 25$ & 222 & $104(46.8)$ & $1.71(0.99,2.98)$ & 0.057 & -1 \\
\hline Age (years) & & & & & 0.714 \\
\hline$<55$ & 552 & $173(31.3)$ & $1.56(1.08,2.24)$ & 0.017 & \\
\hline$\geq 55$ & 360 & $232(64.4)$ & $1.59(0.97,2.59)$ & 0.065 & \\
\hline $\mathrm{TC}(\mathrm{mg} / \mathrm{dL})$ & & & & & 0.239 \\
\hline$<208$ & 451 & $174(38.6)$ & $1.53(1.02,2.30)$ & 0.039 & \\
\hline$\geq 208$ & 461 & $231(50.1)$ & $1.22(0.81,1.83)$ & 0.352 & \\
\hline LDL-C (mg/dL) & & & & & 0.489 \\
\hline$<126$ & 445 & $182(40.9)$ & $1.59(1.11,2.27)$ & 0.011 & \\
\hline$\geq 126$ & 467 & $223(47.8)$ & $1.27(0.81,1.99)$ & 0.290 & \\
\hline eGFR $\left(\mathrm{mL} / \mathrm{min} / 1.73 \mathrm{~m}^{2}\right)$ & & & & & 0.699 \\
\hline$<60$ & 172 & $99(57.6)$ & $1.37(0.75,2.50)$ & 0.310 & \\
\hline$\geq 60$ & 740 & $306(41.4)$ & $1.43(1.05,1.95)$ & 0.022 & \\
\hline Fatty liver & & & & & 0.443 \\
\hline None & 647 & $257(39.7)$ & $1.67(1.19,2.35)$ & 0.003 & \\
\hline Moderate or severe & 265 & $148(55.8)$ & $1.12(0.68,1.84)$ & 0.659 & \\
\hline
\end{tabular}

185 Notes: Adjusted for gender, age, BMI, SBP, DBP, HDL-C, fatty liver, and eGFR. Abbreviations: BMI, body mass

186 index; SBP, systolic blood pressure; DBP, diastolic blood pressure; HDL-C, high-density lipoprotein cholesterol; 
187 eGFR, estimated glomerular filtration rate; TyG, triglyceride glucose index; baPWV, brachial to ankle pulse wave 188 velocity; CI, confidence interval.

\section{Discussion}

190 After controlling for covariates, the results of our population-based cross-sectional study 191 showed a positive and linear association between the TyG index and baPWV as a marker of 192 arterial stiffness in a Japanese population. The results were stable in subgroups according to 193 gender, age, BMI, TC, LDLC, eGFR, and fatty liver. Our findings provide evidence of the 194 substantial role of IR in subclinical atherosclerosis in the general population.

195 Several studies have reported the relationship between the TyG index and baPWV. In a 1-year 196 follow-up study of 366 patients, An et al. reported that IR was an independent predictor of 197 atherosclerotic plaque progression in patients with coronary heart disease [32]. A crosssectional study in China of 823 participants who underwent echocardiographic examination

199 found that the TyG index was not significantly associated with baPWV [33]. In contrast, we 200 found that the TyG index was significantly positively correlated with subclinical atherosclerosis as measured by baPWV. In another cross-sectional study, Li et al. showed that the TyG index was independently and positively associated with baPWV $(\beta=1.02 ; 95 \%$ confidence interval [CI] 0.83-1.20) in hypertensive patients. The participants in the highest TyG quartile had a higher risk of increased baPWV (OR,2.12; 95\% CI,1.80-2.50) after adjusting for age, sex, education, BMI, waist circumference, physical activity, current smoking, current drinking, SBP, DBP, levels of serum uric acid, serum homocysteine, HDL-C, and LDLC, eGFR, self-reported diabetes, antihypertensive drug use and antiplatelet drug use [34]. Moreover, in a cross-sectional including 2,818 Japanese participants, the TyG index showed a good correlation with HOMA-IR (men: $r=0.47$, women: $r=0$ multivariate linear regression analysis (men: $\beta=0.11$, women: $\beta=0.14$ ) and especially with increased high baPWV (men: OR 1.27, 95\% CI 1.07-1.49; women: OR 1.53, 95\% CI 1.16-2.02) in multivariate linear regression analyses. The associations were stronger in women than in men [35]. However, our study results showed no sex differences and the trends did not differ by sex in the subgroup analysis

214 (men: adjusted OR 1.50, 95\% CI 1.07-2.10; women: adjusted OR 1.69, 95\% CI 1.05-2.72; P 215 for interaction=0.693).In another recent study of 2830 elderly Chinese individuals, Zhao et al. 216 reported that the TyG index was significantly associated with arterial stiffness as measured by 217 carotid-femoral pulse wave velocity (cfPWV; OR 1.86, 95\% CI 1.37-2.53), baPWV (OR 1.39, 
for microvascular arteriosclerosis after adjusting for age, sex, BMI, waist circumference, smoking habit, hypertension, family history of premature CVD, diabetes, HDL-C, LDL-C, insulin therapy, and statin therapy [36]. However, we found no significant interaction effect of eGFR and TyG in the subgroup analysis (eGFR $<60 \mathrm{~mL} / \mathrm{min} / 1.73 \mathrm{~m} 2$, adjusted OR $1.37,95 \%$ CI $0.75-2.50 ;$ eGFR $\geq 60 \mathrm{~mL} / \mathrm{min} / 1.73 \mathrm{~m} 2$, adjusted OR $1.43,95 \%$ CI $1.05-1.95, \mathrm{P}$ for interaction=0.699). In a study of 3,587 Korean adults, Lee et al. reported that participants in the highest TyG quartile had a higher risk of arterial stiffness as assessed by baPWV (men: OR 2.92; 95\% CI 1.92-4.44; women: OR 1.84; 95\% CI 1.15-2.96) after adjusting for multiple risk factors [37]. These findings were consistent with the results of our study. In addition, in a study of 2,560 relatively healthy Korean subjects, Won et al. also reported a positive association between TyG index and arterial stiffness $(\beta=0.158)$, consistent with our results. Moreover, our inclusion criteria were more stringent, with an $\mathrm{ABI}<0.95$ to exclude patients with preexisting arteriosclerosis. Furthermore, our stepwise regression analysis showed that TC and LDL-C might influence the correlation between the TyG index and baPWV. Therefore, we divided TC and LDL-C into two parts for subgroup analysis. The results showed that TC, LDL-C, and TyG index had no interaction effect on baPWV (TC <208 mg/dL: adjusted OR 1.53, 95\% CI 1.022.30; TC $\geq 208 \mathrm{mg} / \mathrm{dL}$ : adjusted OR 1.22, 95\% CI 0.81-1.83, P for interaction=0.239; LDL-C $<126 \mathrm{mg} / \mathrm{dL}$ adjusted OR 1.59, 95\% CI 1.11-2.27; LDL-C $\geq 126 \mathrm{mg} / \mathrm{dL}$. adjusted OR 1.27, 95\% CI 0.81-1.99; $\mathrm{P}$ for interaction=0.489). Therefore, the positive association between TyG index and baPWV was robust.

The strengths of this study include: (1) our study had stricter cohort criteria for inclusion by excluding patients with symptomatic coronary artery disease (CAD) and included a broad age range (24-84 years), not merely older persons, as well as $A B I<0.95$. (2) This study is the first to report the relationship between the TyG index and baPWV in Japan. (3) We analyse the TyG index as both continuous and categorical variables to reduce the result contingency and increase the result robustness. (4) In subgroup analysis, likelihood ratio tests and hierarchical linear regression model were applied to confirm modifications and interactions. The results were stable in different subgroups.

247 This study has several additional limitations. First, the cross-sectional design meant that we could not exclude causal relationships based on the findings. Second, the analysis did not include the HOMA-IR index, although this index is the gold standard method for measuring IR. However, previous studies demonstrated a close relationship between HOMA-IR and the 
251 TyG index [39]. In addition, the TyG index is more cost-effective and easier to calculate in

252 routine clinical practice as previous reported[40].

\section{Conclusions}

254 To summarize, the results of this study showed an independent association between the TyG 255 index and arterial stiffness, as measured by baPWV, in a relatively healthy Japanese population 256 after adjusting for conventional factors. These results suggest that IR plays a substantial role

257 in subclinical atherosclerosis and might be an important target for preventing major CV disease.

\section{Data Availability}

259 The original data are freely downloaded at http://www.Datadryad.org/.

\section{Conflict of Interest}

261 The authors declare that there is no conflict of interest regarding the publication of this paper.

\section{Funding Statement}

263 This work was supported by the Specific Research Foundation of Sport of Guangdong Province 264 [No. GDSS2020N80, GDSS2020M006], the Clinical Research Projects of the First Affiliated 265 Hospital of Guangzhou Pharmaceutical University (No. TZDXZX201909) and the Key Realm 266 R\&D Program of Guangdong Province [No.2018B030332001].

\section{Acknowledgments}

268 The authors are grateful for the suggestions and comments from Jie Liu, $\mathrm{PhD}$ (Department of 269 Vascular and Endovascular Surgery, Chinese PLA General Hospital) for reviewing the 270 manuscript, which have been very helpful in improving the manuscript.

\section{$271 \quad$ References}

272 [1] Naghavi M., Abajobir AA., Abbafati C., et al. Global, regional, and national age-sex

273 specific mortality for 264 causes of death, 1980-2016: a systematic analysis for the Global 
Burden of Disease Study 2016. Lancet. 2017;390(10100):1151-1210. doi: 10.1016/S01406736(17)32152-9.

276 [2] Wang L, Zhi F, Gao B, et al. Association between lipid profiles and arterial stiffness: A 277 secondary analysis based on a cross-sectional study. J Int Med Res. 278 2020;48(7):300060520938188. doi: 10.1177/0300060520938188.

279 [3] Nagasato D., Tabuchi H., Masumoto H., et al. Prediction of age and brachial-ankle pulse280 wave velocity using ultra-wide-field pseudo-color images by deep learning. Sci Rep. 2020;10(1):19369. doi: 10.1038/s41598-020-76513-4.

[4] Tsuchikura S., Shoji T., Kimoto E., et al. Brachial-ankle pulse wave velocity as an index of central arterial stiffness. J Atheroscler Thromb. 2010;17(6):658-65. doi: 10.5551/jat.3616. [5] Zhao S, Yu S, Chi C, et al. Association between macro- and microvascular damage and the triglyceride glucose index in community-dwelling elderly individuals: the Northern Shanghai Study. Cardiovasc Diabetol. 2019;18(1):95. doi: 10.1186/s12933-019-0898-x. [6] Matthews DR, Hosker JP, Rudenski AS, et al. Homeostasis model assessment-insulin resistance and beta-cell function from fasting plasma-glucose and insulin concentrations in man. Diabetologia.1985;28(7):412-9. doi: 10.1007/BF00280883.

290 [7] Bonora E, Targher G, Alberiche M, et al. Homeostasis model assessment closely mirrors the glucose clamp technique in the assessment of insulin sensitivity: studies in subjects with various degrees of glucose tolerance and insulin sensitivity. Diabetes Care. 2000;23(1):57-63. doi: 10.2337/diacare.23.1.57.

294 [8] Unger G, Benozzi SF, Perruzza F, et al. Triglycerides, and glucose index: a useful indicator 295 of insulin resistance. Endocrinol Nutr.2014;61(10):533-40. English, Spanish. doi: 10.1016/j.endonu.2014.06.009.

297 [9] Simental-Mendía LE, Rodríguez-Morán M, Guerrero-Romero F. The product of fasting 298 glucose and triglycerides as surrogate for identifying insulin resistance in apparently healthy 299 subjects. Metab Syndr Relat Disord. 2008;6(4):299-304. doi: 10.1089/met.2008.0034.

300 [10] Nor NSM, Lee S, Bacha F, et al. Triglyceride glucose index as a surrogate measure of 301 insulin sensitivity in obese adolescents with normoglycemia, prediabetes, and type 2 diabetes mellitus: comparison with the hyperinsulinemic-euglycemic clamp. Pediatr Diabetes. 2016;17(6):458-65. doi: 10.1111/pedi.12303.

304 [11] Kang B, Yang Y, Lee EY, et al. Triglycerides/ glucose index is a useful surrogate marker 305 of insulin resistance among adolescents. Int J Obesity. 201;41(5):789-792. doi: 306 10.1038/ijo.2017.14. 
IR is more associated with Hypogonadism in Chinese men. Sci Rep. 2017;7(1):15874. doi: 10.1038/s41598-017-16108-8.

310 [13] Locateli JC, Lopes WA, Simões CF, et al. Triglyceride/glucose index is a reliable alternative marker for insulin resistance in South American overweight and obese children and adolescents. J Pediatr Endocrinol Metab. 2019;32(10):1163-1170. doi: 10.1515/jpem-2019-

\section{7.}

314 [14] Cho YR, Ann SH, Won KB, et al. Association between insulin resistance, hyperglycemia,

315 and coronary artery disease according to the presence of diabetes. Sci Rep. 2019;9(1):6129.

316 doi: 10.1038/s41598-019-42700-1.

317 [15] Chiu TH, Tsai HJ, Chiou HC, et al. A high triglyceride-glucose index is associated with

318 left ventricular dysfunction and atherosclerosis. Int J Med Sci. 2021;18(4):1051-1057. doi:

319 10.7150/ijms.53920.

320 [16] Alizargar J, Bai CH, Hsieh NC, et al. Use of the triglyceride-glucose index (TyG) in cardiovascular disease patients. Cardiovasc Diabetol. 2020;19(1):8. doi: 10.1186/s12933-019322 0982-2.

323 [17] Li M, Zhan A, Huang X, et al. Positive association between triglyceride glucose index and arterial stiffness in hypertensive patients: the China H-type Hypertension Registry Study. Cardiovasc Diabetol. 2020;19(1):139. doi: 10.1186/s12933-020-01124-2.

[18] Nakagomi A, Sunami Y, Kawasaki Y, et al. Sex difference in the association between surrogate markers of insulin resistance and arterial stiffness. J Diabetes Complications. 2020;34(6):107442. doi: 10.1016/j.jdiacomp.2019.107442.

[19] Lambrinoudaki I, Kazani MV, Armeni E, et al. The TyG Index as a Marker of Subclinical Atherosclerosis and Arterial Stiffness in Lean and Overweight Postmenopausal Women. Heart Lung Circ. 2018;27(6):716-724. doi: 10.1016/j.hlc.2017.05.142.

332 [20] Munakata M. Brachial-ankle pulse wave velocity in the measurement of arterial stiffness:

333 recent evidence and clinical applications. Curr Hypertens Rev. 2014;10(1):49-57. doi: 10.2174/157340211001141111160957.

335 [21] Huang J, Chen Z, Yuan J, et al. Association Between Body Mass Index (BMI) and 336 Brachial-Ankle Pulse Wave Velocity (baPWV) in Males with Hypertension: A Community337 Based Cross-Section Study in North China. Med Sci Monit. 2019; 25:5241-5257. doi: 338 10.12659/MSM.914881. 
340 Japanese population: a secondary analysis based on a cross-sectional study. Lipids Health Dis. 341 2018;17(1):275. doi: 10.1186/s12944-018-0920-4.

342 [23] Wu S, Jin C, Li S, et al. Aging, Arterial Stiffness, and Blood Pressure Association in 343 Chinese Adults. Hypertension. 2019 Apr;73(4):893-899. doi: 344 10.1161/HYPERTENSIONAHA.118.12396.

345 [24] Zheng M, Zhang X, Chen S, et al. Arterial Stiffness Preceding Diabetes: A Longitudinal 346 Study. Circ Res. 2020;127(12):1491-1498. doi: 10.1161/CIRCRESAHA.120.317950.

347 [25] Fukuda T., Hamaguchi M., Kojima T., et al. Association between serum $\gamma$-glutamyl 348 transpeptidase and atherosclerosis: a population-based cross-sectional study. BMJ Open. 349 2014;4(10): e005413. doi: 10.1136/bmjopen-2014-005413.

350 [26] Hashimoto Y., Hamaguchi M., Kojima T., et al. Modest alcohol consumption reduces the 351 incidence of fatty liver in men: a population-based large-scale cohort study. J Gastroenterol 352 Hepatol. 2015 Mar;30(3):546-52. doi: 10.1111/jgh.12786.

353 [27] Ryu S., Chang Y., Kim DI., et al. gamma-Glutamyl transferase as a predictor of chronic 354 kidney disease in nonhypertensive and nondiabetic Korean men. Clin Chem. 2007;53(1):71-7. 355 doi: 10.1373/clinchem.2006.078980.

356 [28] Hamaguchi M., Kojima T., Itoh Y., et al. The severity of ultrasonographic findings in nonalcoholic fatty liver disease reflects the metabolic syndrome and visceral fat accumulation. Am J Gastroenterol. 2007;102(12):2708-15. doi: 10.1111/j.1572-0241.2007.01526. x.

359 [29] Yamashina A., Tomiyama H., Takeda K., et al. Validity, reproducibility, and clinical 360 significance of noninvasive brachial-ankle pulse wave velocity measurement. Hypertens Res. 2002;25(3):359-64. doi: 10.1291/hypres.25.359.

362 [30] Matsuo S., Imai E., Horio M., et al; Collaborators developing the Japanese equation for 363 estimated GFR. Revised equations for estimated GFR from serum creatinine in Japan. Am J 364 Kidney Dis. 2009;53(6):982-92. doi: 10.1053/j.ajkd.2008.12.034.

365 [31] Simental-Mendía LE, Rodríguez-Morán M, Guerrero-Romero F. The product of fasting 366 glucose and triglycerides as surrogate for identifying insulin resistance in apparently healthy subjects. Metab Syndr Relat Disord. 2008;6(4):299-304. doi: 10.1089/met.2008.0034.

368 [32] An X, Yu D, Zhang R, et al. Insulin resistance predicts progression of de novo 369 atherosclerotic plaques in patients with coronary heart disease: a one-year follow-up study. Cardiovasc Diabetol. 2012; 11:71. doi: 10.1186/1475-2840-11-71. 
[33] Chiu TH, Tsai HJ, Chiou HC, et al. A high triglyceride-glucose index is associated with

372 left ventricular dysfunction and atherosclerosis. Int J Med Sci. 2021;18(4):1051-1057. doi:

373 10.7150/ijms.53920.

374 [34] Li M, Zhan A, Huang X, et al. Positive association between triglyceride glucose index and 375 arterial stiffness in hypertensive patients: the China H-type Hypertension Registry Study.

376 Cardiovasc Diabetol. 2020;19(1):139. doi: 10.1186/s12933-020-01124-2.

377 [35] Nakagomi A., Sunami Y., Kawasaki Y., et al. Sex difference in the association between 378 surrogate markers of insulin resistance and arterial stiffness. J Diabetes Complications. 379 2020;34(6):107442. doi: 10.1016/j.jdiacomp.2019.107442.

380 [36] Zhao S, Yu S, Chi C, et al. Association between macro- and microvascular damage and 381 the triglyceride glucose index in community-dwelling elderly individuals: The Northern 382 Shanghai Cardiovasc Diabetol. 2019;18(1):95. doi: 10.1186/s12933-019-0898-X.

383 [37] Lee SB, Ahn CW, Lee BK, et al. Association between triglyceride glucose index and 384 arterial stiffness in Korean adults. Cardiovasc Diabetol. 2018;17(1):41. doi: 10.1186/s12933385 018-0692-1.

386 [38] Won KB, Park GM, Lee SE, et al. Relationship of insulin resistance estimated by 387 triglyceride glucose index to arterial stiffness. Lipids Health Dis. 2018;17(1):268. doi: 388 10.1186/s12944-018-0914-2.

389 [39] Du T, Yuan G, Zhang M, et al. Clinical usefulness of lipid ratios, visceral adiposity 390 indicators, and the triglycerides and glucose index as risk markers of insulin resistance. 391 Cardiovasc Diabetol. 2014; 13:146. doi: 10.1186/s12933-014-0146-3.

392 [40] En-qian Liu, Ya-ping Weng, Ai-ming Zhou, Chun-lai Zeng, "Association between 393 Triglyceride-Glucose Index and Type 2 Diabetes Mellitus in the Japanese Population: A 394 Secondary Analysis of a Retrospective Cohort Study", BioMed Research 395 International, vol. 2020, 2947067. https://doi.org/10.1155/2020/2947067. 